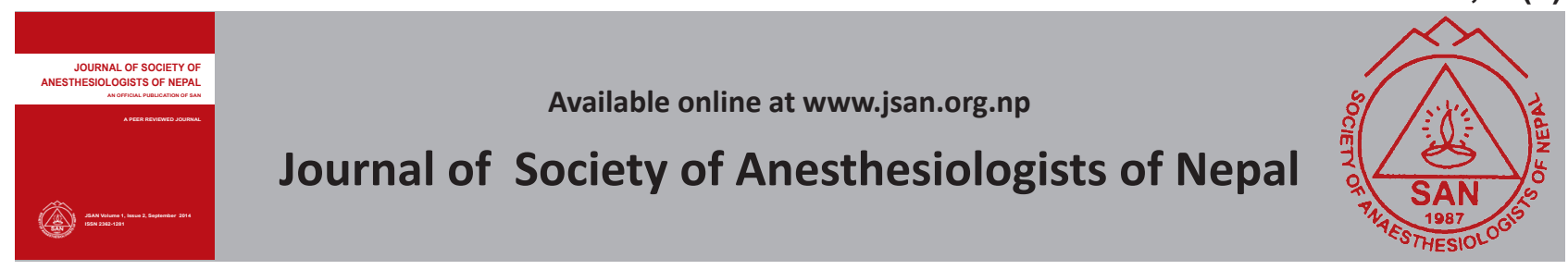

Original Article

\title{
Comparison of postoperative analgesic effectiveness of combined intraperitoneal instillation and periportal infiltration of bupivacaine with intraperitoneal instillation or periportal infiltration alone for laparoscopic cholecystectomy
}

Prajjwal Raj Bhattarai*, Balkrishna Bhattarai**, Birendra Prasad Sah**, Tanvir R Rahman**, Ashish Ghimire**, Shailesh Adhikary***

*Depatrment of Anaesthesiology, Civil Service Hospital, New Baneshwor, Kathmandu, Nepal

**Department of Anaesthesiology \& Critical Care, BPKIHS, Dharan, Sunsari, Nepal

*** Department of Surgery, BPKIHS, Dharan, Sunsari, Nepal

A R T I C L E I N F O
Article history
Received 14.07.2014
Accepted 28.08 .2014
Published 08.09.2014
(C) Authors retain copyright
and grant the journal right
of first publication with the
work simultaneously licensed
under a Creative Commons
Attribution License that allows
others to share the work with
an acknowledgment of the
work's authorship and initial
publication in this journal.

\section{ABSTRACT}

Background: Visceral pain is the major component of pain after laparoscopic cholecystectomy. Periportal infiltration and peritoneal instillation of local anesthetics have been reported to be effective in various laparoscopic procedures. Aim of this study was to find out the analgesic effectiveness and duration of analgesia following combined intraperitoneal instillation and periportal infiltration of bupivacaine and to compare it with peritoneal instillation or periportal infiltration alone.

Methods: A prospective, randomized, double blinded study was conducted in 90 adult patients undergoing elective laparoscopic cholecystectomy. The patients were randomized into three groups. Group 1 received intraperitoneal instillation of $20 \mathrm{ml}$ of $0.5 \%$ bupivacaine. Group 2 received periportal infiltration of $10 \mathrm{ml}$ of $0.25 \%$ bupivacaine. Group 3 received combination of both. After standard balanced anesthesia technique, patients were observed in postoperative period for pain and hemodynamic variables, that were recorded $1 / 2$ hourly for 1 hour, then at 4, 8, 12 and 24 hours postoperatively. The time and doses for rescue analgesia, if given, were also recorded.

Results: Combined peritoneal instillation and periportal infiltration provided a mean duration of analgesia of 6 hours. Peritoneal instillation alone provided analgesia for three hours while periportal infiltration alone provided analgesia for one hour. The combination group required one third (116.67 mg vs. $173.33 \mathrm{mg}$ ) less analgesic than periportal group and one fifth ( $116.67 \mathrm{mg}$ vs. $148.33 \mathrm{mg}$ ) less than that of peritoneal group. No adverse effects were noted.

Conclusion: Combined intraperitoneal instillation and periportal infiltration of bupivacaine reduced postoperative pain after laparoscopic cholecystectomy better than intraperitoneal instillation or periportal infiltration of bupivacaine alone.

Keywords: Analgesia, Bupivacaine, Cholecystectomy, Intraperitoneal injection, Laparoscopic, Pain measurement.

How to cite this article: Bhattarai PR, Bhattarai B, Sah BP, Rahman TR, Ghimire A, Adhikary S. Comparison of postoperative analgesic effectiveness of combined intraperitoneal instillation and periportal infiltration of bupivacaine with intraperitoneal instillation or periportal infiltration alone for laparoscopic cholecystectomy. JSAN 2014;1:59-64.

Corresponding author: Prajjwal Raj Bhattarai, Civil Service Hospital, New Baneshwor, Kathmandu, Nepal

Telephone +9779851165664

E-mail: prajjwal.bhattarai@gmail.com 


\section{Introduction}

Adequate postoperative analgesia has been associated with less physiological derangement and better as well as quicker recovery and ambulation. Various drugs and devices are available to manage post-operative pain effectively, like including non-steroidal antinflammatory drugs, opioids, epidural and spinal analgesia, patient controlled analgesia, local anesthetics and regional or peripheral nerve blocks.' Local anaesthesia techniques are part of the multimodal approach to postoperative pain management. Visceral pain is the major component of postoperative pain after laparoscopic cholecystectomy. Periportal infiltration and peritoneal instillation of local anesthetics have been reported to be effective in various abdominal procedures like laparoscopic cholecystectomy and gynecological laparoscopy for sterilization and diagnosis in addition to open abdominal procedures, such as total abdominal hysterectomy. The rationale for this route of administration is that the peritoneum is exposed to block of visceral nociceptive conduction, thereby providing an additional mechanism of anaesthesia. Absorption from the large peritoneal surface may be a further mechanism of analgesia. This prospective, randomized, double blind trial was designed to assess the effectiveness of intraperitoneal instillation of bupivacaine in combination with periportal infiltration for postoperative analgesia in patients undergoing laparoscopic cholecystectomy. Analgesic effectiveness was compared in terms of Visual Analog Scale (VAS) among intraperitoneal instillation of bupivacaine, local periportal infiltration and intraperitoneal instillation of bupivacaine in combination with periportal infiltration. The duration of analgesia and analgesic sparing effects were also compared.

\section{Methods}

This was a prospective, randomized, double blinded study conducted in 90 patients of ASA physical status (ASA PS) I and II of age group between 16 years to 65 years undergoing routine elective laparoscopic cholecystectomy. Ethical approval obtained from the institutional ethical committee, written and informed consent was obtained from the patients. The patients were divided randomly into three equal groups using computers generated sequence maintained in sequentially numbered opaque envelopes and the study medication was given according to following distribution.

Group 1: - received intraperitoneal instillation of $20 \mathrm{ml}$ of $0.5 \%$ bupivacaine.

Group 2: - received periportal infiltration of $10 \mathrm{ml}$ of $0.25 \%$ bupivacaine.

Group 3: - received combination of periportal infiltration with $10 \mathrm{ml}$ of $0.25 \%$ of bupivacaine and intraperitoneal instillation of $20 \mathrm{ml}$ of $0.5 \%$ of bupivacaine.

Allergy or any contraindication to study medication, conversion of the laparoscopic surgery to open cholecystectomy, patient's refusal to participate in the study and weight less than $40 \mathrm{~kg}$ were used as exclusion criteria.

Medications used in the study involved $0.5 \%$ and $0.25 \%$ of isobaric bupivacaine. For intraperitoneal instillation $20 \mathrm{ml}$ of $0.5 \%$ bupivacaine was used. Drug for periportal injections i.e. $10 \mathrm{ml}$ of $0.25 \%$ bupivacaine was prepared by mixing $5 \mathrm{ml}$ of isotonic saline to $5 \mathrm{ml}$ of $0.5 \%$ bupivacaine. The patients as well as the investigator observing and documenting postoperative analgesia were unaware about the drug used.

\section{Anesthetic technique}

All recruited patients were familiarized and explained about the usage of VAS for pain assessment (zero as "no pain at all" and 10 as "worst imaginable pain") during the preoperative visit. All patients were premeditated with tab diazepam $0.2 \mathrm{mg} / \mathrm{kg}$ given orally in the night before surgery and two hours prior to the surgery. On the day of surgery, in the operation theatre, peripheral venous access was secured and monitors for vital parameters (heart rate, Electrocardiogram, blood pressure, pulse oximeter) were attached. At the same time the randomization was done according to the computer generated sequence.

Anaesthesia was induced with propofol $2 \mathrm{mg} / \mathrm{kg}$, pethidine $1 \mathrm{mg} / \mathrm{kg}$ with vecuronium $0.1 \mathrm{mg} / \mathrm{kg}$ intravenously to facilitate the laryngoscopy and endotrcheal intubation. After endotracheal intubation, anaesthesia was maintained with isoflurane in oxygen with intermittent top ups of vecuronium as required. The study medication was prepared by anesthesia assistant not involved in the study and administered either as intraperitoneal instillation of $20 \mathrm{ml}$ of $0.5 \%$ of bupivacaine with periportal infiltration of $10 \mathrm{ml}$ of $0.25 \%$ of bupivacaine or intraperitoneal instillation $20 \mathrm{ml}$ of $0.5 \%$ bupivacaine alone or periportal infiltration $10 \mathrm{ml}$ of $0.25 \%$ of bupivacaine alone according to randomization before closing the skin incision by the operating surgeons. The intraperitoneal bupivacaine was instilled in trendelenberg position.

Neuromuscular blockade was antagonized by the neostigmine $0.05 \mathrm{mg} / \mathrm{kg}$ and glycopyrrolate $0.01 \mathrm{mg} /$ $\mathrm{kg}$. The trachea was extubated once patient regained the consciousness and patient was transferred to postanaesthesia care unit (PACU). There, patients were observed for pain and other parameters (heart rate, systolic blood pressure and diastolic blood pressure) for one hour before transferring to post-operative ward. No other analgesics were used in the operation theatre or PACU besides study medication till patient complained of pain. The investigator providing anesthetic care as well as the patient were blinded to the study medications. The patient was evaluated in PACU and surgical ward by the same investigator. The surgeon and the nursing staff were also kept unaware about the group allocation. VAS and other study parameters were recorded $1 / 2$ hourly for 1 hour 
in the PACU then in the surgery ward at 4 hours, 8 hours, 12 hours and 24 hours postoperatively. The time and doses for rescue analgesia, if given, were also recorded.

\section{Assessment and management of pain}

Pain intensity was measured using visual analogue scale (VAS). VAS ruler used consisted of a $10 \mathrm{~cm}$ horizontal line, with 'no pain' at one end (end zero) and 'worst imaginable pain' at other end (end 10). The intensity of pain was assessed by asking the patients to grade the severity of pain by marking on the $10 \mathrm{~cm}$ line scale at the point that corresponded to the level of pain they felt. The distance from patient's mark in centimeter from no pain (end zero) in whole numbers was taken as numerical index of the severity of pain. If any patient complained of pain immediately after extubation of trachea, the patient was considered to have a pain of VAS more than four and was managed accordingly. If the severity of pain (VAS) was more than four, the patient was given intravenous injection tramadol $50 \mathrm{mg}$ slowly as rescue analgesic and was repeated whenever required. Time of administration of rescue analgesic was noted and total amount of the drug consumed during study period was also noted. Time of administration of first dose of rescue analgesic was considered as time of termination of post-operative analgesic effect of study medication. Blood pressure, pulse and respiratory rate along with the VAS, at the time points mentioned above, were noted in post anaesthesia care unit and post-operative ward. Any sign of systemic toxicity or hypersensitivity reaction or other adverse effects were treated accordingly and noted.

\section{Statistical analysis}

The obtained data was compiled in Excel worksheet software and analyzed using Statistical Package for Social Science (SPSS17.0). Results were compared using student t-test for the continuous data and contingency table for categorical data. Mean values among the groups were compared with one-way ANOVA test. A p-value of 0.05 or less was considered statistically significant. After completion of data analysis, decoding of the group was done for interpretation.

\section{Results}

In this study a total of 90 patients, undergoing surgery for laparoscopic cholecystectomy under general anaesthesia were enrolled. The patients were between age group of 20 years to 61 years and had ASA physical status of I and II. Demographic parameters, duration of surgery and anesthesia were comparable between the groups (Table 1).
Table 1: Comparison of demographic parameters, duration of surgery and anesthesia

\begin{tabular}{|c|c|c|c|c|}
\hline \multirow[t]{2}{*}{ Variable } & \multicolumn{3}{|l|}{ Group } & \multirow[t]{2}{*}{ p-value } \\
\hline & $1(n=30)$ & $2(n=30)$ & $3(n=30)$ & \\
\hline $\begin{array}{l}\text { Mean Age in } \\
\text { years (SD) }\end{array}$ & $\begin{array}{l}39.33 \\
(10.97)\end{array}$ & $\begin{array}{l}39.00 \\
(11.08)\end{array}$ & $\begin{array}{l}37.23 \\
(10.89)\end{array}$ & 0.727 \\
\hline $\begin{array}{l}\text { Sex } \\
\text { ratio(Female/ } \\
\text { Male) }\end{array}$ & $23 / 7$ & $24 / 6$ & $27 / 3$ & 0.496 \\
\hline $\begin{array}{l}\text { Mean Weight in } \\
\text { kg (SD) }\end{array}$ & $\begin{array}{l}54.33 \\
(6.26)\end{array}$ & $\begin{array}{l}54.73 \\
(5.98)\end{array}$ & $\begin{array}{l}55.67 \\
(4.36)\end{array}$ & 0.640 \\
\hline ASA- PS (I/II) & $14 / 16$ & $17 / 13$ & $16 / 14$ & 0.739 \\
\hline $\begin{array}{l}\text { Mean duration } \\
\text { of }\end{array}$ & $\begin{array}{l}107.8 \\
(27.18)\end{array}$ & $\begin{array}{l}102.33 \\
(17.35)\end{array}$ & $\begin{array}{l}109.67 \\
(28.19)\end{array}$ & 0.492 \\
\hline $\begin{array}{l}\text { Anaesthesia in } \\
\text { minutes(SD) }\end{array}$ & & & & \\
\hline $\begin{array}{l}\text { Mean duration } \\
\text { of surgery } \\
\text { in minutes(SD) }\end{array}$ & $\begin{array}{l}94.50 \\
(23.97)\end{array}$ & $\begin{array}{l}89.67 \\
(18.56)\end{array}$ & $\begin{array}{l}95.83 \\
(29.33)\end{array}$ & 0.589 \\
\hline
\end{tabular}

SD - Standard Deviation

\section{POSTOPERATIVE ANALGESIA}

The parameters observed included VAS for pain, time to the first dose of analgesia and 24 hours analgesic requirements.

\section{Visual analog Scale (VAS)}

Significant differences in VAS were observed at 30 minutes, 60 minutes, 240 minutes and 480 minutes postoperatively among the three groups. The values thereafter were comparable (figure 1). At 30 minutes postoperatively, the mean (SD) VAS Scores was 1.50 (0.82) in group 1, 4.43 (1.59) in group 2 and $0.83(0.73)$ in group 3 . The value in group 2 was significantly higher than group 1 and $3(p<0.05)$. At 60 minutes the mean (SD) VAS Scores was 1.93 (1.48) in group 1, 3.53 (1.96) in group 2 and 1.57 (1.63) in group 3 $(p<0.05)$. At 480 minutes the mean (SD) VAS Scores was $2.47(1.29)$ in group $1,3.07$ (1.50) in group 2 and 1.50(1.07) $(p<0.05)$. 


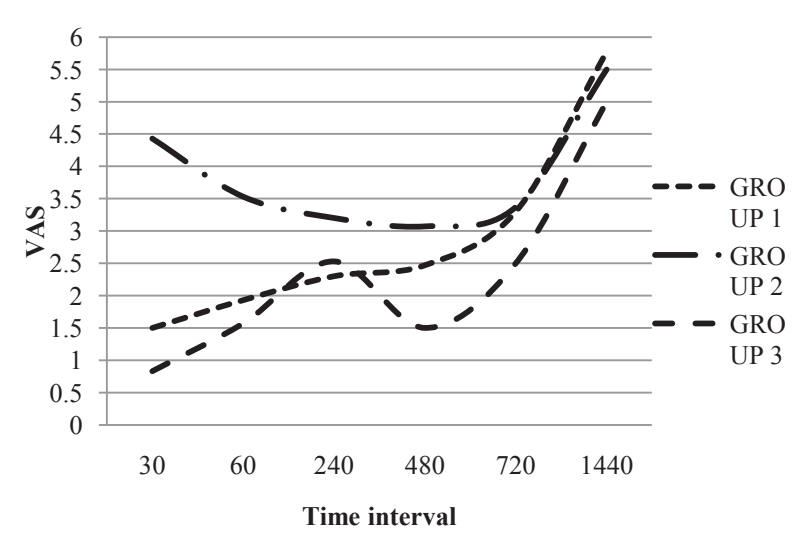

Fig 2.1 : Comparison of VAS Score

Wide and significant differences were observed in time to the first dose of analgesia (duration of analgesia) and the total 24 hours analgesic requirement (Figure 2) The median values were more striking i.e. 195 minutes (Interquartile Range $\{I Q R\}=60-270), 30$ minutes (IQR=30-76.25) and 450 minutes (IQR=195-510) in group1, 2 and 3 respectively.

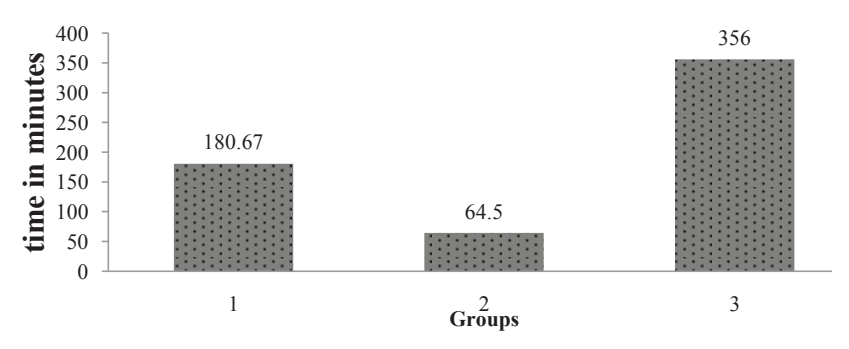

Figure 2: Comparison of time for the first dose of rescue analgesia

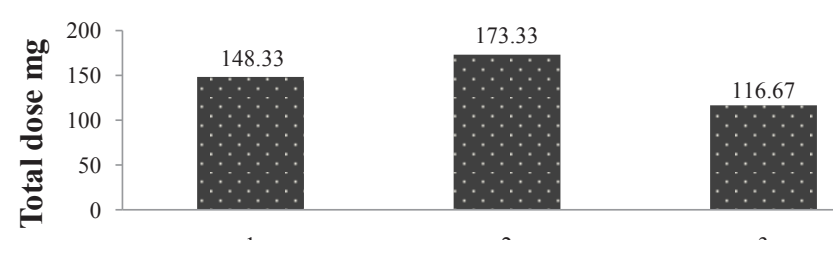

Fig 3: Comparison of total dose of rescue analgesia consumption

There was wide variation in 24 hours analgesic requirements among all three groups (Figure 3) Group 2 required the highest total dose and group 3 the least $(p=0.001)$.

\section{VITAL PARAMETERS}

The vital hemodynamic parameters were assessed at intervals of 30 minutes, 60 minutes, 240 minutes, 480 minutes, and 720 minutes and 1440 minutes. The pulse rate recorded was lowest at 60 minutes in all groups while it was the highest at 240 minutes in group 1 and 2 and 1440 minutes in group 3 . The pulse rate at all the time points were comparable among all the groups except at 480 minutes when the difference between group 1 and 3 was statistically significant $(p=0.004)$.

The respiratory rate remained significantly lower in group 3 than group 1 and 2 in all the observations except at 1440 minutes. The lowest respiratory rates were observed in all the 3 groups at 1440 minutes. In group 1, the highest respiratory rate was observed at 60 minutes and that in group 2 was at 240 minutes. In group 3, respiratory rate was high at 30 and 60 minutes, it was significantly lower than other two groups.

The systolic and diastolic blood pressures recorded at the given time points were comparable among all the three groups. In all groups, the lowest systolic and diastolic blood pressures were observed at 60 minutes postoperatively. The highest blood pressures in group 1 and 2 were observed at 240 minutes and that in group 3 was observed at 1440 minutes.

\section{Discussion}

In the present study, the analgesic effects of intraperitoneal instillation and periportal infiltration of bupivacaine on postoperative pain in patients undergoing laparoscopic cholecystectomy were investigated. For this study, we chose patients undergoing laparoscopic cholecystectomy only. The procedure is less complicated, is minimally invasive, without significant tissue damage and has less variation in performance time and technique. The postoperative pain induced by this type of surgery has been found to have a considerable visceral component ${ }^{4}$, (owing to surgical handling and diaphragmatic irritation by dissolved carbon dioxide) and a lesser component that is somatic in origin (owing to the holes made in the abdominal wall for the trocars).

Although marked individual variability of pain is characteristic after laparoscopic cholecystectomy, ${ }^{7}$ the reasons for this significant variability are not clear. Consistent with many previous studies, the main finding of our study was effective analgesia for seven hours as well as significant reduction in analgesic requirement for 24 hours postoperatively without remarkable adverse effects following intraperitoneal instillation of $0.5 \%$ bupivacaine in combination with periportal infiltration.

In the present study we chose $0.5 \%$ concentration of bupivacaine for peritoneal instillation based on the reports that this concentration is effective in producing analgesia as well as that the plasma concentration is not likely to exceed the safe limit." Further, the total amount of drug used in peritoneal instillation and periportal infiltration does not exceed the safe limit of $3 \mathrm{mg} / \mathrm{kg}$ of bupivacaine when patients weight of less than $40 \mathrm{~kg}$ are excluded.

Present study has shown that combination of intraperitoneal instillation and periportal infiltration provides longer and more effective analgesia as compared to intraperitoneal instillation or periportal infiltration 
alone. Combination provided effective analgesia for mean duration of six hours with median duration of seven and half hours, which is double the duration provided by intraperitoneal instillation alone. The shortest duration of pain free time postoperatively was found in patients receiving periportal infiltration alone with mean duration of about one hour and median of half an hour.

Reduction in VAS scores up to eight hours and Verbal Rating Scale (VRS) up to four hours with the use of intraperitoneal instillation of bupivacaine was observed in the study conducted by Bhardwaj et al. This finding can be considered close to our finding. Much longer duration of analgesia up to 48 hours has been reported by Narchi and colleagues $^{8}$ following intraperitoneal instillation of local anesthetics in patients undergoing diagnostic laparoscopy. Similarly remarkable analgesia was noted by Pasqualucci and colleagues. They used intraperitoneal instillation of local anesthetic in patients undergoing diagnostic laparoscopy where injury to the visceral tissue is very minimal.

In contrast, Rademaker and coworkers failed to demonstrate reduction in postoperative pain with instillation of 20 $\mathrm{ml}$ of $0.25 \%$ bupivacaine or $0.5 \%$ lignocaine in patients undergoing laparoscopic cholecystectomy. Evidently they had used local anesthetic in lower concentration which probably is the reason for their contrasting finding. Moreover, they had instilled the drug in supine position without any degree of Trendelenberg position which also probably prevented the accumulation of the drug in the intended site i.e. gall bladder bed.

Postoperative VAS scores in our study also clearly indicate the analgesic effects of the intervention. Patients receiving combined intraperitoneal instillation and periportal infiltration had significantly lower VAS scores as compared to patients receiving periportal infiltration alone for eight hours. Patients receiving intraperitoneal instillation alone had significantly lower VAS scores compared to patients receiving periportal infiltration alone for four hours.

In fact the VAS scores in patients receiving periportal infiltration alone had lower VAS scores than in the combination groups at four hours observation. At 12 hours and thereafter no difference was observed in VAS among the groups. This pattern of VAS scores observed in our study can be attributed to the fact that the observation taken near the time of peak effect of already administered dose of analgesic are naturally likely to be relatively lower.

In the studies conducted by Bhardwaj $\mathrm{N}$ et $\mathrm{al}^{11}$ and Pasqualucci et $\mathrm{al}^{12}$, lower pain scores have been observed for relatively shorter duration in VRS but with much longer duration in VAS. However, there are some differences between theirs and our study. They have added adrenaline to the bupivacaine and they have not used periportal infiltration as in our case. Similar reduction in pain intensity has been observed with the intraperitoneal use of ropivacaine in patients undergoing laparoscopic cholecystectomy. In another study by Maharjan et al, combined peritoneal instillation and periportal infiltration used for postoperative pain reduction has been reported for longer duration than ours. But there are numbers of differences between their and our studies as they used different volume and concentration of drug, they had smaller sample size and the study design was also different.

The 24 hours postoperative analgesic requirement in our study clearly indicated effectiveness of combination of peritoneal instillation and periportal infiltration compared to peritoneal instillation or periportal infiltration alone. The combination group required one third less analgesic dose than the periportal infiltration group and more than one fifth less than peritoneal instillation group. On analyzing various reported literature we can see variation in the reduction in the postoperative analgesia requirements achieved with intraperitoneal instillation and periportal infiltration. ${ }^{5,8,10,11,13}$ But this cannot be considered unexpected as every investigator team has its own study design, technique and method of pain relief.

It is interesting to note that vital parameters observed in our study have indirectly but clearly substantiated our finding of postoperative analgesia. One can observe least variation of the vital parameter in the combination group as compared to other two groups. Our finding support the fact that visceral pain as well as somatic pain accounts for the major discomfort experienced in early postoperative period. The pain relief was very short lived or ineffective in patients receiving periportal infiltration as compared to the patients receiving peritoneal instillation.

In conclusion, findings of our study show that combined intraperitoneal and periportal infiltration of bupivacaine reduces postoperative pain after laparoscopic cholecystectomy better than intraperitoneal instillation or periportal infiltration of bupivacaine alone without causing hemodynamic instability and adverse effects. Administrating bupivacaine intraperitoneally and periporatlly is an inexpensive, effective, easy to administer and relatively safe method as a part of a multi-modal approach to control post-operative pain after laparoscopic cholecystectomy.

\section{Limitation of the study}

Patient-controlled analgesia with other titrable opioids would have been better option than bolus tramadol for rescue analgesia. The outcome variables and hemodynamic parameters were observed periodically rather than continuously or more frequently. Individualization of the dose i.e. dose of bupivacaine calculated per unit body weight could not be done.

Acknowledgements The authors acknowledge all the staff of operating room, PACU, postoperative ward for giving us access to information.

Conflict of Interest Statement. The authors have no potential, perceived, or real conflicts of interests. 


\section{References}

1. Jensen $\mathrm{K}$, Kehlet $\mathrm{H}$. Post-operative recovery profile after laparoscopic cholecystectomy a prospective, observational study of a multimodal anesthetic regime. Acta Anaesthesiol Scand. 2007;51:464-71.

2. Salerno A, Hermann R. Efficacy and safety of steroid use for postoperative pain relief: update and review of medical literature. J Bone Joint Surg Am 2006;1361-72.

3. Michaloliakou C, Chung F, Sharma S. Preoperative multimodal analgesia facilitates recovery after ambulatory laparoscopic cholecystectomy. Anesth Analg 1996;82:44-51.

4. Joris J, Thiry E, Paris P. Pain after laparoscopic cholecystectomy characteristics and effect of intraperitoneal bupivacaine. Anesth Analg 1995;81:379-84.

5. Moiniche $\mathrm{S}$, Jorgensen $\mathrm{H}$, Wetterslev J. Local anesthetic infiltration for postoperative pain relief after laparoscopy a qualitative and quantitative systematic review of intraperitoneal, port-site infiltration and mesosalpinx block. Anesth Analg 2000;90:899-912.

6. Ure BM, Troidl H, Spangenberger W. Pain after laparoscopic cholecystectorny. Surg Endosc 1994;8:90-6.

7. Narchi P, Benhamaou D, Bouaziz H. Serum concentrations of local anaesthetics following intraperitoneal administration during laparoscopy. Eur J Clin Pharmacol 1992;42:223-5.

8. Raetzell M, Maier C, Schroder D. Intraperitoneal application of bupivacaine during laparoscopic cholecystectomy risk or benefit. Anesth Analg 1995;81:967-72.

9. Scheinin B, Kellokumpu I, Lindgren L. Effect of intraperitoneal bupivacaine on pain after laparoscopic cholecystectomy. Acta Anesthesiol Scand 1995;39:195-8.

10. Bhardwaj N. Intraperitoneal bupivacaine instillation for Postoperative pain relief after laparoscopic Cholecystectomy. Indian J. Anaesth 2002;46:49-52.

11. Pasqualucci A, Angelis V, Contardo R. Pre-emptive analgesia: Intraperitoneal local anesthetic in laparoscopic cholecystectomy. Anaesthesiology 1996;85:11-20.

12. Rademaker BM, Ringers J, Odoom JA. Pulmonary function a stress response after laparoscopic cholecystectomy: comparison with subcostal incision and influence of thoracic epidural anaesthesia. Anesth and Analg 1992;75:381-5.

13. Pappas-Gogos G, Tsimogiannis KE. Preincisional and intraperitoneal ropivacaine plus normal saline infusion for postoperative pain relief after laparoscopic cholecystectomy. Surg endosc 2008;22:2036-45.

14. Maharjan SK, Shrestha S. Intraperitoneal and periportal injection of bupivacaine for pain after laparoscopic cholecystectomy. Kathmandu Univ Med J 2009;7:50-3. 